UCRL-JC-128289

PREPRINT

\title{
Multilayer reflective coatings for extreme-ultraviolet lithography
}

\author{
C. Montcalm, S. Bajt, P. B. Mirkarimi, \\ E. Spiller, F. J. Weber, and J. A. Folta
}

This paper was prepared for and presented at the 23rd Annual International Symposium on Microlithography

Santa Clara, California

February 22-27, 1998

March 10, 1998

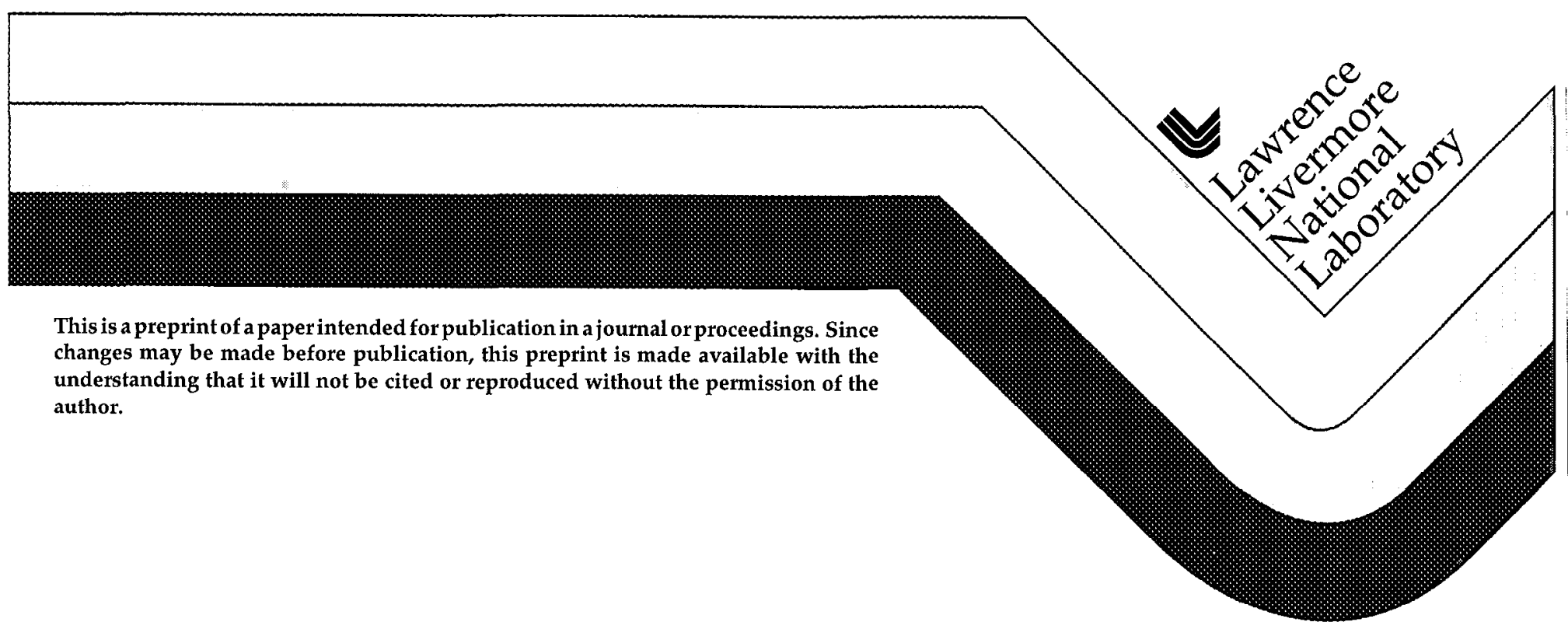




\section{DISCLAIMER}

This document was prepared as an account of work sponsored by an agency of the United States Government. Neither the United States Government nor the University of California nor any of their employees, makes any warranty, express or implied, or assumes any legal liability or responsibility for the accuracy, completeness, or usefulness of any information, apparatus, product, or process disclosed, or represents that its use would not infringe privately owned rights. Reference herein to any specific commercial product, process, or service by trade name, trademark, manufacturer, or otherwise, does not necessarily constitute or imply its endorsement, recommendation, or favoring by the United States Government or the University of California. The views and opinions of authors expressed herein do not necessarily state or reflect those of the United States Government or the University of California, and shall not be used for advertising or product endorsement purposes. 


\title{
Multilayer reflective coatings for extreme-ultraviolet lithography
}

\author{
Claude Montcalm, ${ }^{\dagger}$ Saša Bajt, Paul B. Mirkarimi, Eberhard Spiller, Frank J. Weber, and James A. Folta \\ Advanced Microtechnology Program \\ Lawrence Livermore National Laboratory \\ P.O. Box 808, L-395, Livermore, California 94550.
}

\begin{abstract}
Multilayer mirror coatings which reflect extreme ultraviolet (EUV) radiation are a key enabling technology for EUV lithography. $\mathrm{Mo} / \mathrm{Si}$ multilayers with reflectances of $67.5 \%$ at $13.4 \mathrm{~nm}$ are now routinely achieved and reflectances of $702 \%$ at 11.4 $\mathrm{nm}$ were obtained with $\mathrm{Mo} / \mathrm{Be}$ multilayers. High reflectance is achieved with careful control of substrate quality, layer thicknesses, multilayer materials, interface quality, and surface termination. Reflectance and film stress were found to be stable relative to the requirements for application to EUV lithography. The run-to-run reproducibility of the reflectance peak position was characterized to be better than $0.2 \%$, providing the required wavelength matching among the seven multilayercoated mirrors used in the present lithography system design. Uniformity of coating was improved to better than $0.5 \%$ across $150 \mathrm{~mm}$ diameter substrates. These improvements in EUV multilayer mirror technology will enable us to meet the stringent specifications for coating the large optical substrates for our next-generation EUV lithography system.
\end{abstract}

Keywords: Extreme ultraviolet (EUV) lithography, reflective coatings, multilayers, reflectance, stress, radiation stability, sputtering deposition, uniformity, reproducibility.

\section{INTRODUCTION}

The present consensus design for an industrially viable extreme-ultraviolet (EUV) projection lithography system incorporates multiple reflective optics: (1) four condenser optics to collect EUV radiation from the source and illuminate the mask; (2) a reflective mask consisting of an integrated circuit metalization pattern on a multilayer mirror; and (3) four precision projection optics to project a reduced image of the mask onto a resist-coated wafer. ${ }^{1}$ Seven of the nine surfaces operate at near-normal incidence and require coatings with EUV multilayer mirrors. The EUV projection lithography system throughput is therefore a strong function of the multilayer reflectance because of the seven reflection design.

The multilayer coatings typically consist of alternating layers of molybdenum and silicon $(\mathrm{Mo} / \mathrm{Si})$ or molybdenum and beryllium $(\mathrm{Mo} / \mathrm{Be})$, where the thicknesses of each layer are determined by the trade-off between maximizing the constructivc interference of the beams reflected at each interface and minimizing the overall absorption to enable more interfaces to contribute to the reflectance. ${ }^{2}$ The resulting multilayer structures resemble a quarter-wave stack in which the rclative thickness of the Mo layers in the bilayer has been slightly reduced. This results in individual thicknesses of $2.8 \mathrm{~nm}$ for Mo and $4.1 \mathrm{~nm}$ for $\mathrm{Si}$ in the $\mathrm{Mo} / \mathrm{Si}$ case, or $2.3 \mathrm{~nm}$ for $\mathrm{Mo}$ and $3.4 \mathrm{~nm}$ for $\mathrm{Be}$ in the $\mathrm{Mo} / \mathrm{Be}$ case.

The multilayer mirrors must meet stringent requirements for a production EUV projection lithography system to be practical in terms of performance and throughput. Initial lithography system throughput analysis and cost-of-ownership estimates have led us to set goals for multilayer mirrors with $70 \%$ or higher reflectance which is stable for $3-5$ years. ${ }^{3}$ Five years ago, the $\mathrm{Mo} / \mathrm{Si}$ multilayers had already proven to be effective reflective coatings for normal incidence in the 13.0$13.5 \mathrm{~nm}$ wavelength region with reflectances around $65.5 \%$ being achieved routinely. ${ }^{4} \mathrm{Mo} / \mathrm{Be}$ multilayers had been much less investigated but reflectances around $68 \%$ at normal incidence were reported in the 11.1-11.5 wavelength region. ${ }^{5}$ With

$\dagger^{\dagger}$ C. Montcalm (correspondence): E-mail: montcalm@ Ilnl.gov; Telephone: 925-424-2903; Fax: 925-422-8761 
the likelihood of better reflectances in the near future, it is no exaggeration to claim that these developments in reflective multilayer technology have enabled EUV lithography.

However, several issues other than the multilayer reflectance were then raised. The precision projection optics must be fabricated with aspheric surface figures that are accuratc to within $0.25 \mathrm{~nm}$ to maintain diffraction-limited performance. ${ }^{6}$ This dictates a rigorous control on the thickness distribution of the deposited multilayers and an accurate knowledge of the multilayer stress since they both affect the final figure of the optics. Also, since these multilayers mirrors have fairly narrow bandpasses, about $0.56 \mathrm{~nm}$ for $\mathrm{Mo} / \mathrm{Si}$, the reflectance peak positions of the mirrors must be all aligned to within $0.05 \mathrm{~nm}$ to preserve at least $90 \%$ of the optimum throughput. This means that the multilayer coating on each element must have the same bilayer thickness to within an accuracy of $0.025 \mathrm{~nm}$. This imposes an unprecedented control on the run-to-run reproducibility of the deposition system and an accurate knowledge of the multilayer stability as a function of temperature and radiation since the mirrors are not all exposed to the same environment.

This manuscript describes our current status of knowledge on the reflectivity, the stress and the stability of the Mo/Si and $\mathrm{Mo} / \mathrm{Be}$ multilayer mirrors (Section 2). It also discusses some of the recent developments in multilayer mirror technology which make the realization of advanced optical designs for the EUV projection lithography system possible (Section 3). Finally, it reviews remaining issues of the multilayer technology that must be solved to enable fabrication of EUV lithography production tools (Section 4).

\section{MULTILAYER DEVELOPMENT}

The three key issues related to the material properties of EUV multilayer mirrors are reflectance, stress and stability; with reflectance having the most importance. In the following sections we treat each of these issues for both the $\mathrm{Mo} / \mathrm{Si}$ and the $\mathrm{Mo} / \mathrm{Be}$ multilayer mirrors.

\subsection{Normal incidence reflectance}

The EUV projection lithography system throughput is a strong function of the multilayer reflectance because of the seven reflection design. ${ }^{4}$ It is therefore critical that the multilayer mirrors have the highest possible normal incidence reflectance. $\mathrm{Mo} / \mathrm{Si}$ and $\mathrm{Mo} / \mathrm{Be}$ are today's material pairs of choice due to both their superior optical properties in the EUV wavelength region and their structural properties on the sub-nanometer scale. They are the only material pairs for which multilayer mirrors with normal incidence reflectances higher than $60 \%$ were achieved in the EUV spectral region. 7,8

Figure 1 shows the reflectance versus wavelength curves of the best $\mathrm{Mo} / \mathrm{Si}$ and $\mathrm{Mo} / \mathrm{Be}$ multilayer mirrors produced to date in our laboratory. The measurements were made at $5^{\circ}$ from normal incidence using the EUV reflectometer at the Lawrence Berkeley National Laboratory (LBNL) which is attached to beamline 6.3.2 of the Advanced Light Source (ALS). ${ }^{9}$ The reflectance curve of the Mo/Si multilayer reaches a peak value of $67.5 \%$ at $13.42 \mathrm{~nm}$ while the $\mathrm{Mo} / \mathrm{Be}$ multilayer has a peak reflectance of $70.2 \%$ at $11.34 \mathrm{~nm}$. The spectral bandwidth of these mirrors, measured at full width at half-maximum (FWHM), is $0.56 \mathrm{~nm}$ and $0.27 \mathrm{~nm}$ for the $\mathrm{Mo} / \mathrm{Si}$ and $\mathrm{Mo} / \mathrm{Be}$ multilayer mirrors, respectively. These multilayers were both deposited on standard $\mathrm{Si}<100>$ substrates with DC-magnetron sputtering using the deposition systems described in Section 3.1. The Mo/Si multilayer consists of 40 bilayers with a period thickness of $6.89 \mathrm{~nm}$ and a thickness ratio, i.e., the relative Mo layer thickness, of 0.37 . The $\mathrm{Mo} / \mathrm{Be}$ multilayer consists of 70 bilayers with a period thickness of $5.74 \mathrm{~nm}$ and a thickness ratio of 0.42 . The Mo/Si multilayer has a Si layer as the top-most layer on the surface while the $\mathrm{Mo} / \mathrm{Be}$ multilayer has a $\mathrm{Be}$ top layer.

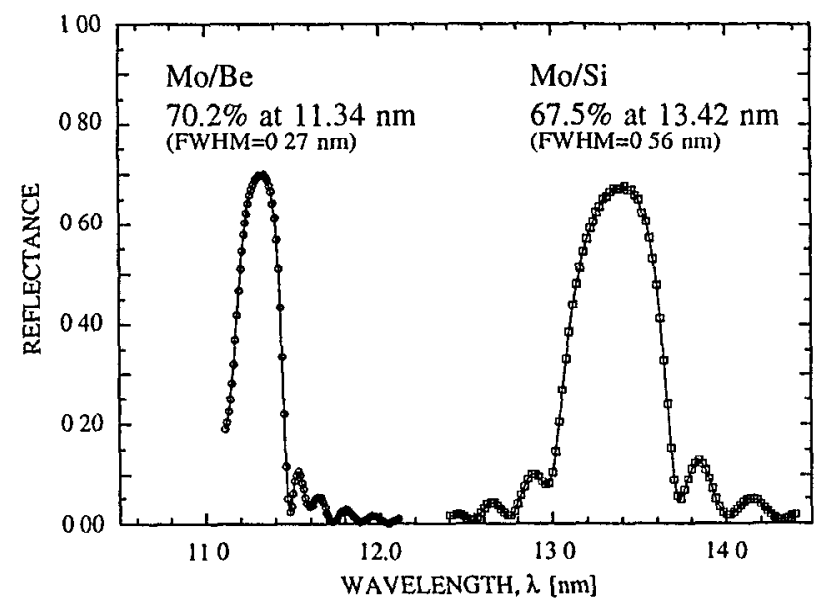

Figure 1. Reflectance response of typical $\mathrm{Mo} / \mathrm{Si}$ and $\mathrm{Mo} / \mathrm{Be}$ multilayer mirror coatings measured at $5^{\circ}$ from normal incidence. Peak reflectances of $70.2 \%$ and $67.5 \%$ are routinely achieved for $\mathrm{Mo} / \mathrm{Be}$ and $\mathrm{Mo} / \mathrm{Si}$, respectively. 
It is possible to simulate these reflectance curves and extract information concerning the limitations that prevent higher peak reflectances from being achieved. For example, Fig. 2 shows the measured reflectance curve of the $\mathrm{Mo} / \mathrm{Si}$ multilayer mirror shown in the previous figure (circles), together with the best theoretical fit to the data (solid line) and the theoretical reflectance of an ideal or perfect multilayer structure (dotted curve). Also shown in this figure is the calculated reflectance curve taking only the interface roughness into account (dash-dotted curve). The reflectance calculations were made using a standard recursive method 10,11 with optical constants derived from scattering factors taken from the literature. ${ }^{12}$ The best fit to the data was obtained assuming linear interface width parameters ${ }^{13}$ (roughness) of $0.6 \mathrm{~nm}$ and $0.3 \mathrm{~nm}$ for the Mo-on-Si and Si-on-Mo interfaces, respectively, and a $4.5 \mathrm{~nm}$ thick oxide layer $\left(\mathrm{SiO}_{2}\right)$ on the surface of the multilayer. A similar fit to the $\mathrm{Mo} / \mathrm{Be}$ multilayer reflectance curve of Fig. 1 can be obtained using interface widths of $0.4 \mathrm{~nm}$ and $0.2 \mathrm{~nm}$ for the Mo-on-Be and Be-on-Mo interfaces, respectively, and a $3.0 \mathrm{~nm}$ thick oxide layer $(\mathrm{BeO})$ on the surface of the multilayer.

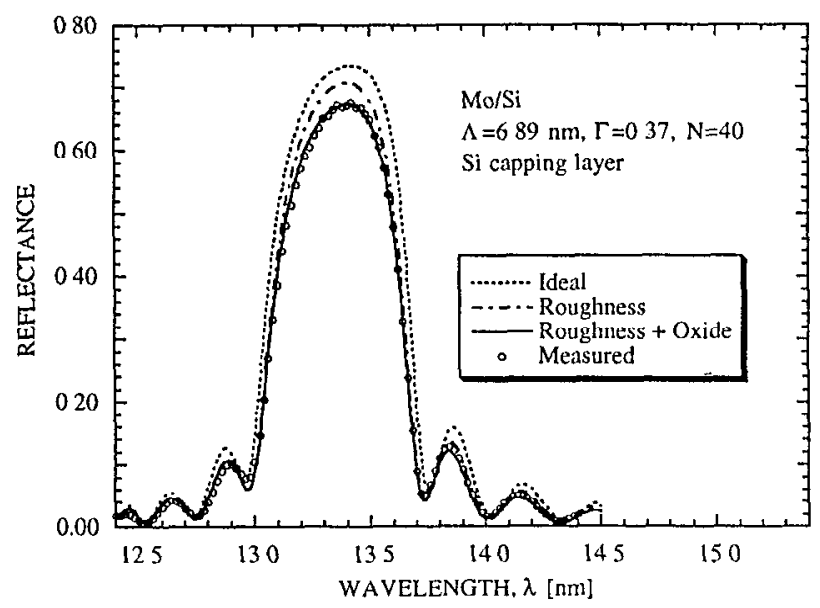

Figure 2. Measured reflectance of a typical Mo/Si multilayer compared to the calculated reflectances for an ideal multilayer, a multilayer with rough interfaces, and a multilayer with rough interfaces and an oxidized surface.

The model identifies the two major limitations to achieving the highest possible peak reflectances in a Mo/Si or a $\mathrm{Mo} / \mathrm{Be}$ multilayer mirror. The theoretical peak reflectance of an ideal multilayer is $\sim 75 \%$ for the $\mathrm{Mo} / \mathrm{Si}$ multilayer mirrors and $\sim 80 \%$ for the Mo/Be multilayer mirrors. However, the existence of diffuse interlayers of mixed composition at the layer boundaries limits the reflectance of each interface ${ }^{4,14}$ and the existence of a contamination or corrosion layer on the surface of the multilayer ${ }^{15}$ also significantly reduces the reflectance. For the Mo/Si multilayers, not much can be done with magnetron sputtering to further minimize the formation of the interlayer at the Mo-Si interfaces since the deposition conditions have been optimized exhaustively. Improvements may be possible, however, if the multilayers are deposited with ion beam sputtering since this technique offers more control on the energetics of the layer growth. For the Mo/Bc multilayers, significant improvement can still be expected since research in Mo/Be multilayer is relatively new.

Concerning the second limitation, the formation of a contamination layer at the surface of the multilayer, there is definitely room for improvement. The problem was first reported by Underwood et al ${ }^{15}$ where the reflectance drop versus exposure time to air was measured for two Mo/Si multilayer mirrors, one with a Mo layer as the top layer and the other with a top $\mathrm{Si}$ layer. Although the sample with the top Si layer had a lower peak reflectance at the beginning, its performance remained unchanged after several months of exposure to air while the performance of the sample with the top Mo layer significantly decreased. When the Mo layer was exposed directly to air, it eventually became completely oxidized to $\mathrm{MoO}_{3}$ and $\mathrm{MoO}_{2}$ and contaminated with carbonaceous materials causing a 10 to $12 \%$ decrease in absolute reflectance. The oxidation of $\mathrm{Si}$ is not as severe but still forms approximately 3 to $5 \mathrm{~nm}$ thick $\mathrm{SiO}_{2}$ which causes a 4 to $5 \%$ decrease in reflectance. For the $\mathrm{Mo} / \mathrm{Be}$ multilayers, we have found that it is preferable to have $\mathrm{Be}$ as the capping layer rather than Mo, showing once again that Mo strongly oxidizes. This suggests that reflectance increases $(>2 \%)$ should be possible if a thin passivating layer of a non-reactive material (e.g., carbon) is deposited on the surface of the multilayer before exposure to air. The mechanical modifications necessary to deposit such an overcoat layer have been implemented in our magnetron sputtering deposition systems. Also, a theoretical investigation of candidate capping materials has recently been performed. Figure 3 shows the predicted reflectance improvements for different capping layers on an ideal Mo/Si multilayer with Si as the layer underneath the capping layer. Note, however, that the calculated curves shown in Fig. 3 are optimistic since they neglect the fact that these materials may also get contaminated or adsorb water vapor to some extent.

Finally, multilayer reflectance strongly depends on the high-spatial-frequency (HSF) roughness of the optical substrate. HSF substrate roughness not only degrades the smoothness of the multilayer interfaces, but more importantly, it causes high angle scatter of the EUV radiation. ${ }^{16,17}$ Figure 4 shows the decreasing reflectance of Mo/Si multilayers deposited on zerodur substrates with increasing HSF roughness. The roughness was determined from $2 \mu \mathrm{m}$ square atomic force microscopy (AFM) scans. Our typical substrates used for multilayer development-both fused silica optical flats and prime grade Si $\langle 100\rangle$ wafers-have surface finishes which are routinely better than $0.1 \mathrm{~nm} \mathrm{rms}$. Advanced EUV lithography system designs 


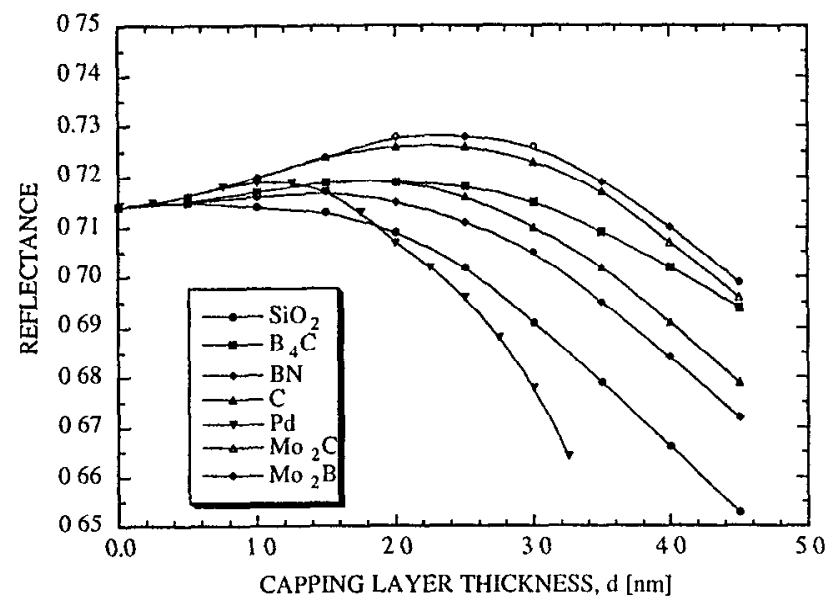

Figure 3. Calculated peak reflectances for ideal Mo/Si multilayers with a variety of increasingly thicker capping layer materials on top of the last Si layer.

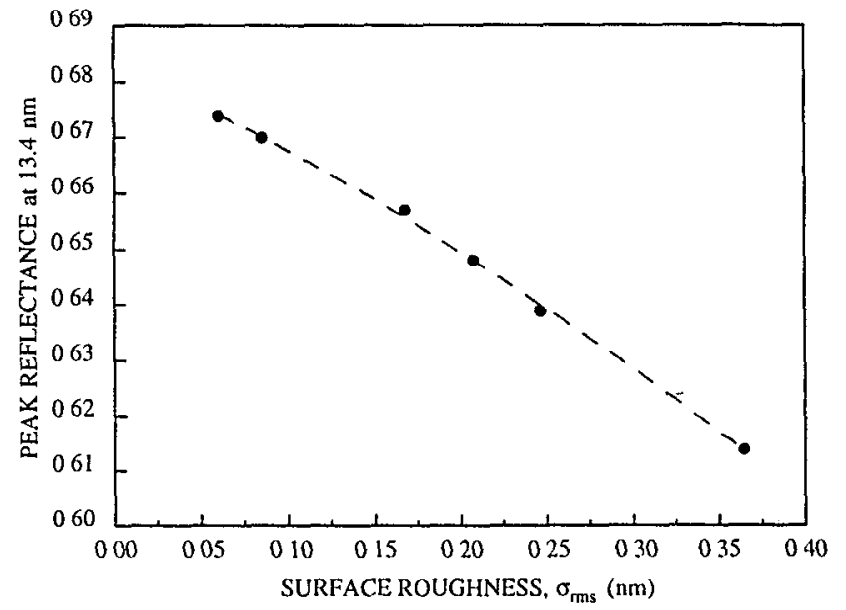

Figure 4. The effect of substrate roughness upon multilayer peak reflectance. Substrates were Zerodur flats with varying levels of surface roughness. Roughness values shown correspond to the high spatial frequency component as measured by atomic force microscopy on $2 \mu \mathrm{m}$ square scans.

require aspheric optical substrates with extremely tight specifications on aspheric figure and both mid- and high- spatial frequency roughness. However much recent progress has been achieved in fabricating ultra-polished aspheric optics and a clear development path for the required substrates has been identified. ${ }^{6}$

\subsection{Residual stress}

The EUV multilayers optimized for reflectance typically have a stress of $-420 \mathrm{MPa}$ (compressive) for Mo/Si and $+330 \mathrm{MPa}$ (tensile) for $\mathrm{Mo} / \mathrm{Be} .^{18}$ This level of film stress is large enough to deform the figure of the projection optics in an EUV lithography system. ${ }^{19}$ Due to the stringent surface figure requirements for these optics, it is desirable to minimize deformation, and in particular non-spherical deformation, of the optics due to the multilayer film stress. Except near the edges, most of the deformation due to film stress should be spherical. It is expected that the spherical component of the deformation will be compensated by adjustment of the position of the optics during alignment; however, such methods may not compensate for some of the non-spherical component of the deformation. 19

Previous techniques for reducing stress in $\mathrm{Mo} / \mathrm{Si}$ multilayer films included varying the composition, varying the base pressure, and post-deposition annealing. ${ }^{20-28}$ However, any technique used to reduce the effects of multilayer film stress must do so without incurring a significant loss in reflectance, since the reflectance has a strong impact on the throughput of an EUV lithography tool. We have tested these methods and found that post-deposition annealing yields the greatest stress reduction at the lowest cost in reflectance. ${ }^{18}$ For example, the stress can be reduced by $75 \%$ with only a $1.3 \%$ (absolute) drop in reflectance at annealing temperatures near $200{ }^{\circ} \mathrm{C}$. However, since it has not been determined if the precisely figured substrates can withstand a $200^{\circ} \mathrm{C}$ anneal, it was decided that to reduce risk a viable non-thermal (athermal) technique for stress reduction should also be developed.

Using an athermal buffer-layer technique with amorphous silicon and Mo/Be buffer-layers it was possible to obtain $\mathrm{Mo} / \mathrm{Be}$ and $\mathrm{Mo} / \mathrm{Si}$ multilayers with a near-zero net film stress and less than a $1 \%$ loss in reflectance. ${ }^{18}$ For example a Mo/Be multilayer with $68.7 \%$ reflectance at $11.4 \mathrm{~nm}$ and a Mo/Si multilayer with $66.5 \%$ reflectance at $13.3 \mathrm{~nm}$ were produced with net stress values of less than $30 \mathrm{MPa}$.

\subsection{Temporal and radiation stability}

It is important that the EUV reflectance be stable over time and under EUV irradiation. The temporal stability of the reflectance and peak wavelength of a $\mathrm{Mo} / \mathrm{Si}$ multilayer with a reflectance of approximately $66.4 \%$ was monitored over a period of 18 months (Fig. 5). The reflectance (circles) remained stable and the fluctuation in the measured value indicates a relative uncertainty of $0.5 \%$ in the measurements. The reflectance peak position slightly shifted, however, from $13.16 \mathrm{~nm}$ to 


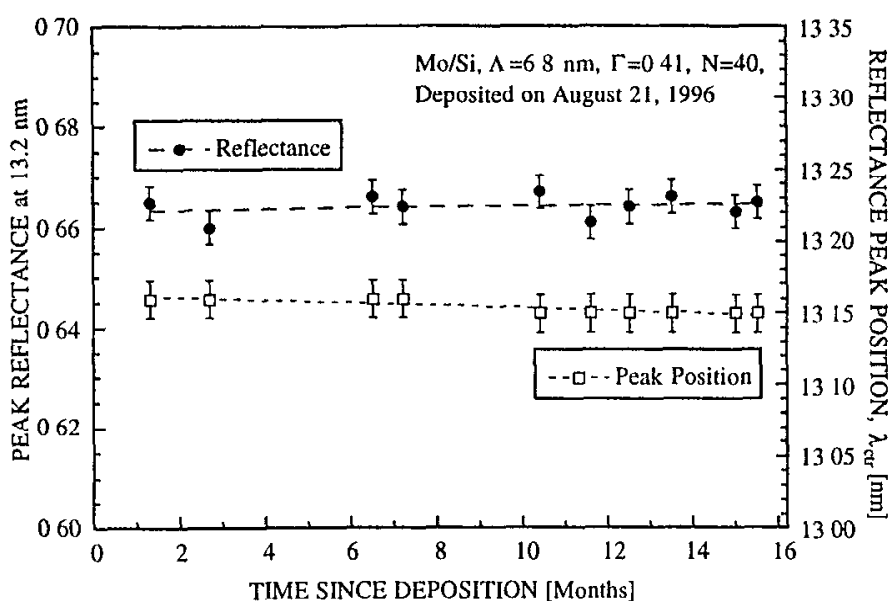

Figure 5. Temporal stability of peak reflectance and peak wavelength for a typical Mo/Si multilayer.

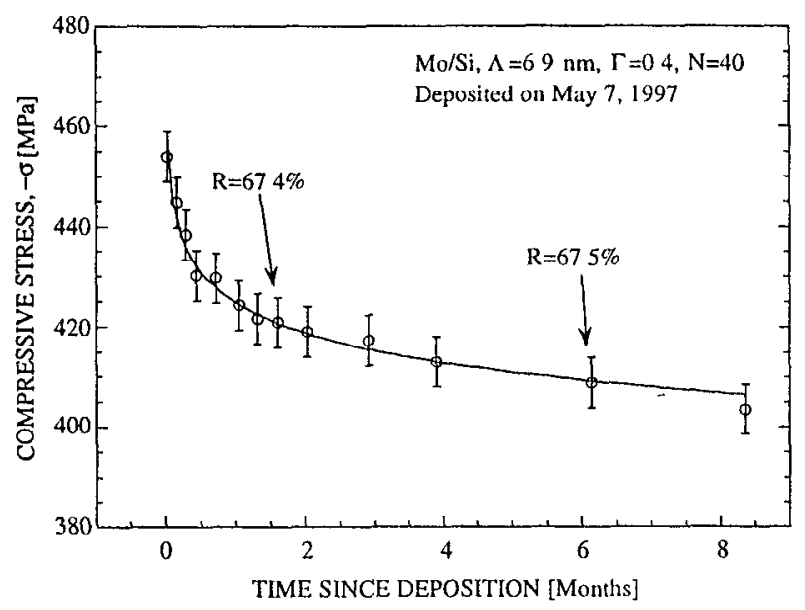

Figure 6. Temporal stability of stress for a typical $\mathrm{Mo} / \mathrm{Si}$ multilayer. After the initial relaxation period of about 10 days, subsequent stress changes are not expected to affect the performance of an EUV lithography system.

$13.15 \mathrm{~nm}$. This small shift is indicative of a bilayer thickness contraction which can most likely be attributed to a slow silicide formation at the interfaces. ${ }^{29} \mathrm{We}$ recently began monitoring the stress (and reflectance) of another Mo/Si multilayer which had as-deposited stress of $-455 \mathrm{MPa}$. As shown in Fig. 6, the stress was observed to decrease significantly (7\%) during the first month, but changed by only $4 \%$ thereafter, which is not a problem for EUV lithography systems. ${ }^{19}$

It is also important that the multilayer mirrors maintain high reflectance after exposure to radiation typical of an EUV lithography system. The stability of high reflectance $\mathrm{Mo} / \mathrm{Si}$ multilayers under exposure to EUV radiation has been studied by several researchers. However, the study undertaken in 1992 by Gaines et al..$^{30}$ is the only reported work where the incident spectrum and dose were carefully matched to the spectrum and dose incident on the first imaging optic of an EUV lithography camera system. In addition, this was the only reported work where the temperature was carefully controlled to eliminate effects due to thermal annealing. No measurable degradation in reflectance was measured; hence there is some assurance that radiation damagc should not affect the first imaging mirror in an EUV lithography camera system after one year of operation. However the report did not examine the radiation damage susceptibility of the first stage condenser optics which receive not only much higher doses of EUV radiation, hut also high doses of UV, visible, and IR radiation.

Although we believe the multilayers are stable against the radiation flux present in EUV lithography systems, we are currently performing more exhaustive radiation stability experiments in which we increase the exposure dose by a factor of 10 to $100 x$ over previous experiments.

\section{DEPOSITION TECHNOLOGIES}

As shown in the previous sections, the current state-of-the-art EUV multilayer mirrors are of sufficient quality to be used in a commercial EUV lithography system. However, several challenges must be overcome in deposition technology for these coatings to be deposited with the thickness control necessary to meet the stringent specifications over the large substrates required.

\subsection{Deposition system and procedure}

Our Mo/Si and Mo/Be multilayers are currently deposited in two similar DC-magnetron sputtering systems (Fig. 7). ${ }^{31}$ The primary difference between the $\mathrm{Mo} / \mathrm{Si}$ and $\mathrm{Mo} / \mathrm{Be}$ deposition systems is that the $\mathrm{Mo} / \mathrm{Be}$ system is housed in an exhausted enclosure to prevent exposure to toxic Be dust. The systems contain two $12.7 \times 25.4 \mathrm{~cm}$ rectangular sputter sources placed $180^{\circ}$ apart, i.e., at diametrically opposite sides of a circular chamber, with chimneys that limit the deposition region to the area directly above them. Two substrates located $90^{\circ}$ apart are held face down on a rotating table (platter) at a distance of $6 \mathrm{~cm}$ above the sources in a "sputter-up" configuration. The sputter chamber is typically cryo-pumped until the pressure is in 


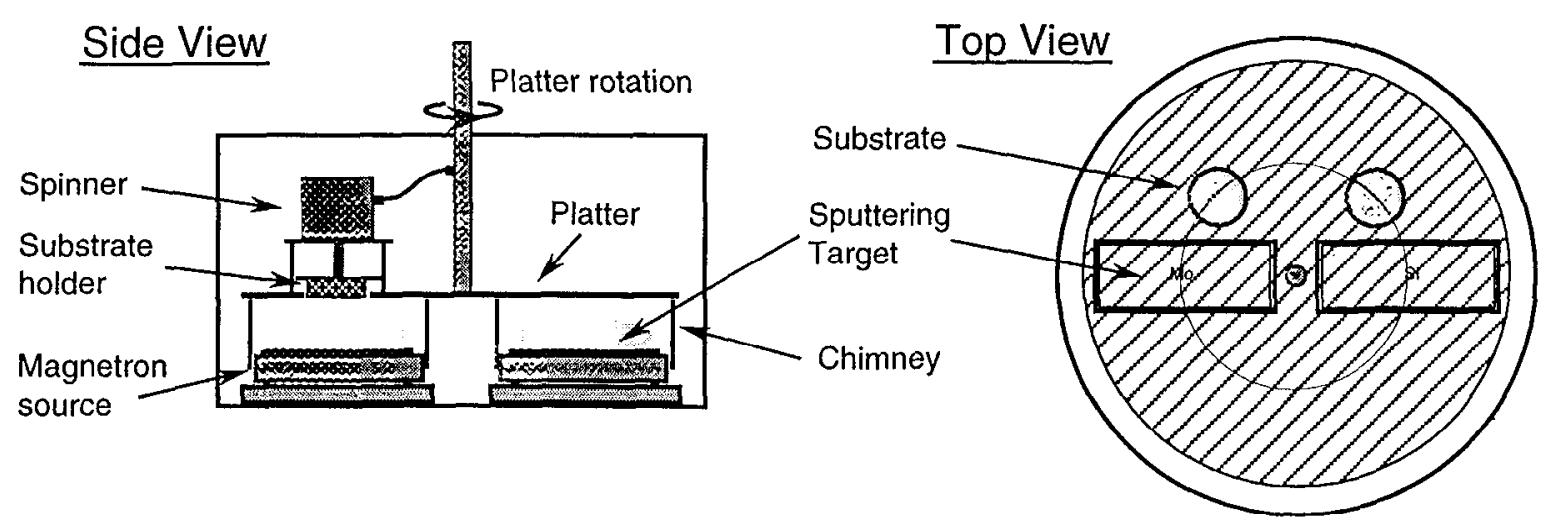

Figure 7. Layout of the DC magnetron sputter system used to deposit multilayer coatings.

the $10^{-8}$ Torr range. Ultrahigh purity Ar at pressure of $0.50-2.00$ mTorr is used to sputter the $\mathrm{Si}$, Mo and $\mathrm{Be}$ targets at source powers of $100-400 \mathrm{~W}$.

The multilayers are deposited by sweeping the substrates above the sources with a controlled rotation of the platter. A bilayer is deposited with each complete revolution of the platter. The layer thicknesses are determined by the time the substrate is exposed to the source which, in turn, depends on the substrate transit velocity. Additionally, the substrates are spun around their axis of symmetry which provides better azimuthal uniformity by averaging out the spatial variation of the deposition rates. The arrangement of the substrates and the sputter sources is such that at any given time only one substrate is over a source and being coated. Therefore, the two substrates can be coated with identical or completely different (and independent) multilayer structures, which is very useful in several comparative studies.

\subsection{Uniform multilayer coatings}

To preserve the figure of the projection optics, thickness control to within $0.5 \%$ will be necessary across an optical substrate $150 \mathrm{~mm}$ in diameter. This imposes a rigorous control on the uniformity of the multilayer period thickness over the surface of the substrate.

The relative thickness variation of our $\mathrm{Mo} / \mathrm{Si}$ multilayers is about $6 \%$ over a $150 \mathrm{~mm}$ diameter region when the deposition is made by rotating the platter at constant velocity (Fig. 8). We can improve the uniformity of multilayer thickness by use of a platter velocity profiling technique in which the platter revolution speed is varied as a function of its position relative to the sources. The optimum velocity profile is determined by an iterative approach. In our case, the peripheral coating thickness obtained from a constant velocity rotation is too thin. Therefore, the substrate is moved more slowly while the periphery is cntering and leaving the deposition zoncs. In this manner, variation in coating thickness was reduced from $5.2 \%$ to $0.4 \%$ peak-to-valley over a $140 \mathrm{~mm}$ diameter region on flat $\mathrm{Si}$ substrates.

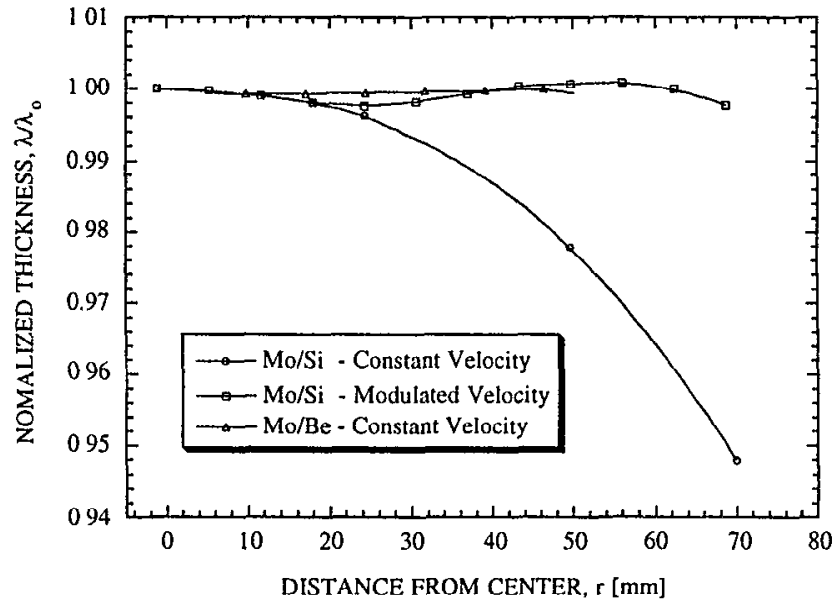

Figure 8. Spatial uniformity of Mo/Si multilayer film thickness has been improved from $5.2 \%$ peak-to-valley to better than $0.4 \%$ over a $140 \mathrm{~mm}$ diameter region on flat Si substrates. $\mathrm{Mo} / \mathrm{Be}$ multilayers have $0.1 \%$ uniformity over $100 \mathrm{~mm}$ diameter.

Also shown in Fig. 8 is the thickness uniformity of our Mo/Be multilayers when deposited with a constant platter rotation velocity. In this case, the uniformity is much better, within $0.1 \%$ peak-to-valley, due to an improved design of the sputtering sources. Indeed, with these sources the magnetic field is such that the erosion profile on the sputtering target is much more uniform resulting in a more uniform flux distribution of the deposition. 

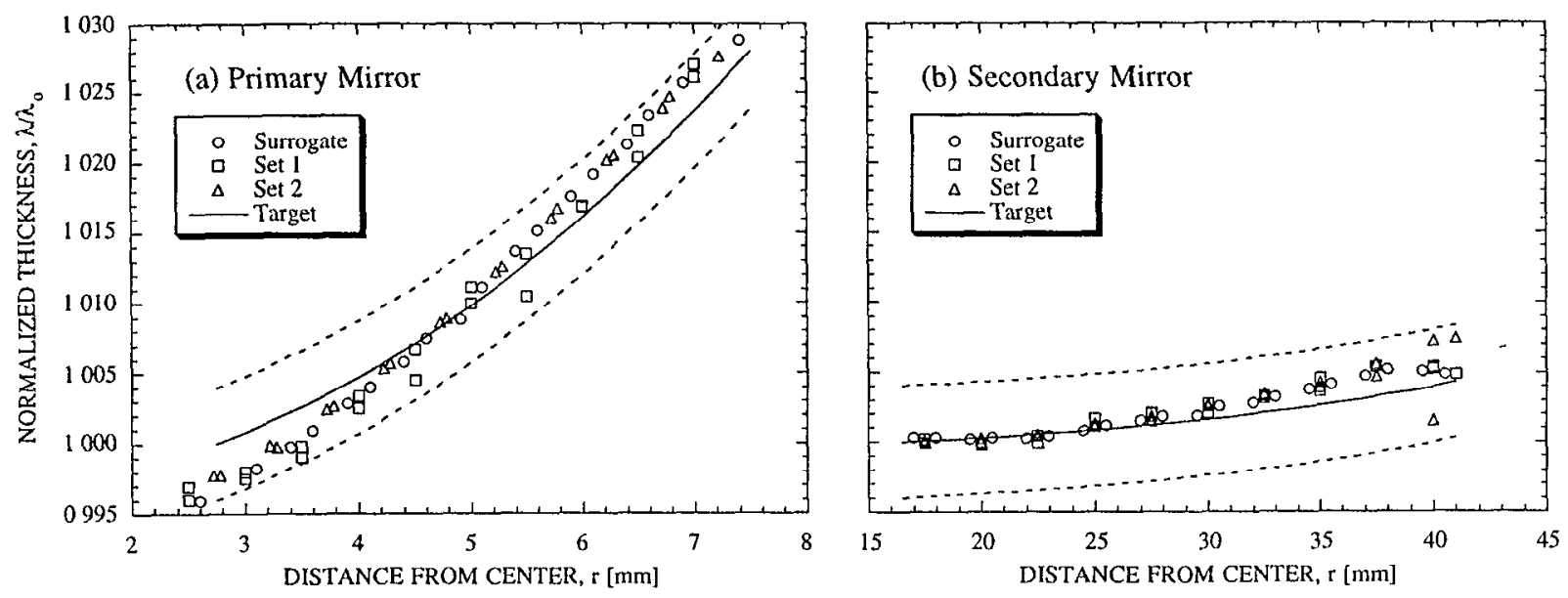

Figure 9. Thickness gradient of multilayers deposited on a 10x Schwarzschild camera for a prototype EUV lithography system. Figures $9 \mathrm{a}$ and $9 \mathrm{~b}$ represent the primary and secondary mirrors of the Schwarzschild system, respectively. The solid line is the target value, the dashed lines represent the boundaries of the specifications, and the symbols are the measured values for three subsequent depositions.

\subsection{Graded multilayer coatings}

Some optics require multilayer coatings with a thickness gradient in order to accommodate a variation in angle of incidence of the EUV radiation. A prime example is the first condenser optic which must collect a large solid angle of the radiation from the laser plasma source. ${ }^{1}$ Other examples are the optics of the EUV 10x microstepper systent, i.e., the two Schwarzschild projection optics and the ellipsoidal condenser optic. ${ }^{32}$

We have coated the mirrors for two sets of Schwarzschild projection and condenser optics for the 10x microstepper system. ${ }^{33}$ The tolerances for these optics were about $\pm 0.1 \mathrm{~nm}$, which represent $\pm 0.75 \%$ of the EUV reflectance peak position $(13.4 \mathrm{~nm})$. We obtained the desired thickness gradient by using a shadow mask in front of each of the spinning optics, while the absolutc valuc of the thickness was adjusted with the rotation speed of the platter. The shape of each shadow mask was obtained in several ( 2 to 3 ) iterations using data from a previous mask to derive the required change in mask shape. The mask-making iterations were stopped when the deviations from the desired profile were less than $\pm 0.4 \%$ peak. Figures $9 \mathrm{a}$ and $9 \mathrm{~b}$ show the resulting thickness profiles for the two projection optics for three consecutive deposition runs. The three sets of data in each plot show that we can reproduce the thickness profile from one deposition run to another within a $0.1 \%$ error when the same mask is used. This is about a factor of 8 better than the present specifications. The absolute value of the multilayer thickness, however, showed larger variations from run-to-run with a standard deviation of $0.08 \mathrm{~nm}$, just within the allowed tolerances. It will be necessary to reduce these variations if we want to make use of the excellent reproducibility of the profile data.

\subsection{Wavelength matching}

Run-to-run repeatability of deposition rate is critical to insure that multiple optics in a system operate at the same peak wavelength. Wavelength-mismatched optics reduce the optical throughput of a system with multiple reflections. Figure 10 shows how the throughput of a 7 reflection system is reduced as the standard deviation of the optic-to-optic mismatch increases. The reflectance-wavelength response curves were assumed to have a gaussian distribution. We

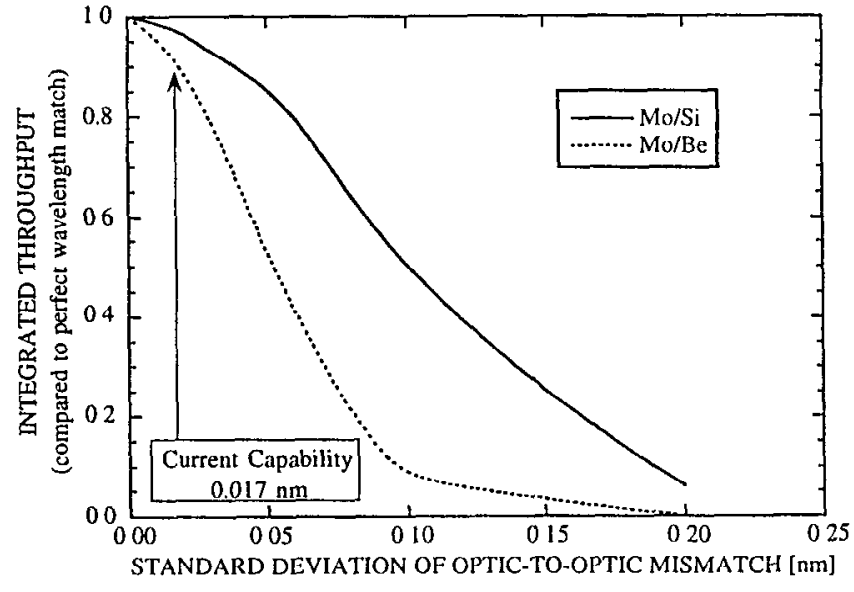

Figure 10. Optical throughput loss expected from optic-tooptic mismatch of peak wavelength for an optical system with 7 multilayer mirrors. The narrower bandpass of $\mathrm{Mo} / \mathrm{Be}$ mirrors $(0.27 \mathrm{~nm}$ FWHM) results in higher loss than for $\mathrm{Mo} / \mathrm{Si}$ $(0.54 \mathrm{~nm}$ FWHM). We currently achieve wavelength matching of $0.017 \mathrm{~nm}$ at 1 standard deviation. 
have measured the standard deviation of our run-to-run repeatability of peak wavelength to be $0.017 \mathrm{~nm}$, or $0.13 \%$ on flat $\mathrm{Si}$ wafers. According to Fig. 10, this results in an optical throughput loss of $4 \%$ for $\mathrm{Mo} / \mathrm{Si}$ and $10 \%$ for $\mathrm{Mo} / \mathrm{Be}$. $\mathrm{Mo} / \mathrm{Be}$ suffers a greater throughput loss for the same level of process repeatability because the narrower bandpass of $\mathrm{Mo} / \mathrm{Be}$ (FWHM $=0.27 \mathrm{~nm} v s .0 .56 \mathrm{~nm}$ for $\mathrm{Mo} / \mathrm{Si}$ ) amplifies the effect of mismatching.

\section{REMAINING ISSUES}

An increasing part of our work is in the multilayer coating of relatively large optics to demonstrate the commercial viability of high throughput EUV lithography. These optics will be aspherical, some will be deeply concave, and will require either highly uniform or accurately graded coatings. We are retrofitting our existing deposition system as well as designing new deposition systems to meet the challenges of coating large optics-for both existing and future advanced designs of EUV lithography systems.

The high cost of the optical substrates makes it desirable to have techniques available for substrate recovery-that is, the ability to either remove or overcoat a multilayer which does not meet performance specifications. Multiple approaches for substrate recovery are possible, including wet etching, dry etching and simply coating over the original multilayer.

\section{CONCLUSION}

Multilayer mirror technology has advanced to meet most of the stringent requirements of extreme-ultraviolet (EUV) lithography. Near-normal incidence $\left(5^{\circ}\right)$ reflectances of $67.5 \%$ at $13.4 \mathrm{~nm}$ are now routinely achieved with Mo/Si multilayers; while a reflectance of $70.2 \%$ at $11.4 \mathrm{~nm}$ was obtained with $\mathrm{Mo} / \mathrm{Be}$ multilayers. Methods to reduce or counteract the stress of these two multilayer systems with minimal loss of reflectance were developed. The reflectance and stress of the as-deposited multilayers were also found to be relatively stable after initial time periods of 10 days to 40 days for the reflectance or the stress, respectively.

In addition to these material science related multilayer developments, several improvements in the deposition technology were made. High reflectance coatings can now be deposited with layer thickness control better than $0.5 \%$ across substrates up to $150 \mathrm{~mm}$ in diameter. The run-to-run reproducibility of our deposition system, which is essential to obtain near-perfect wavelength matching among the different elements of the EUV lithography camera, was characterized to be better than $0.2 \%$. The primary remaining challenge lies in applying our high performance coatings to the large optics (up to $270 \mathrm{~mm}$ diameter) needed to demonstrate EUV lithography.

\section{ACKNOWLEDGMENTS}

The authors would like to thank R. D. Behymer, F. R. Grabner, M. A. Schmidt and G. B. Wells for their assistance in the maintenance and operation of the multilayer deposition systems. The authors also acknowledge the support of E. M. Gullikson and J. H. Underwood in the EUV reflectance measurements at the LBNL and S. L. Baker in the AFM measurements. Finally, it should be noted that most of the multilayer development work at the LLNL has originally been done by D. G. Stearns, S. P. Vernon and D. P. Gaines. The authors are especially grateful to them for sharing their experience throughout numerous enlightening discussions. This work was performed under the auspices of the U.S. Department of Energy by the Lawrence Livermore National Laboratory under Contract No. W-7405-ENG-48. Funding was provided by the Extreme Ultraviolet Limited Liability Company (EUV LLC) under a Cooperative Research and Development Agreement.

\section{REFERENCES}

1. D.W. Sweeney, D.R. Shafer, H.N. Chapman, and R.M. Hudyma, "EUV optical design for a $0.1 \mu \mathrm{m}$ imaging system," in these proceedings.

2. E. Spiller, Soft X-Ray Optics (SPIE Optical Engineering Press, Bellingham, 1994). 
3. A.M. Hawryluk and N.M. Ceglio, "EUV lithography cost of ownership analysis," in Vol. 23 of OSA Proceedings on Extreme Ultraviolet Lithography series, F. Zernike and D.T. Atwood, Eds. (Optical Society of America, Washington, D.C., 1995), p. 13.

4. D.G. Stearns, R.S. Rosen, and S.P. Vernon, "Multilayer mirror technology for soft-x-ray projection lithography," Appl. Opt. 32, 6952-6960 (1993).

5. K.M. Skulina, C.S. Alford, R.M. Bionta, D.M. Makowiecki, E.M. Gullikson, R. Soufli, J.B. Kortright, and J.H. Underwood, "Molybdenum/beryllium multilayer mirrors for normal incidence in the extreme ultraviolet," Appl. Opt. 34, 3727-3730 (1995).

6. J.S. Taylor, G.E. Sommargren, D.W. Sweeney, and R.M. Hudyma, "The fabrication and testing of optics for EUV lithography," in these proceedings.

7. A tabulation of all the best reported reflectances is available at the following web site: http://wwwcxro.lbl.gov/multilaycr/survey.html

8. Peak reflectances near $66 \%$ at $13.4 \mathrm{~nm}$ have recently been achieved with $\mathrm{Mo}_{2} \mathrm{C} / \mathrm{Si}$ multilayers deposited by $\mathrm{T}$.W. Barbee, Jr., Lawrence Livermore National Laboratory (unpublished results).

9. J.H. Underwood, E.M. Gullikson, M. Koike, P.J. Batson, P.E. Denham, K.D. Franck, R.E. Tackaberry, and W.F. Steele, "Calibration and standards beamline 6.3.2 at the Advanced Light Source," Rev. Sci. Instrum. 67, (1996). Available on CD only.

10. H.A. Macleod, Thin-Film Optical Filters, 2nd ed. (Mc Graw-Hill, New York, 1989).

11. M. Born and E. Wolf, Principles of Optics, 6th ed. (Pergamon Press, New York, 1980).

12. B.L. Henke, E.M. Gullikson, and J.C. Davis, "X-ray interactions: photoabsorption, scattering, transmission, and reflection at $E=50-30,000 \mathrm{eV}, \mathrm{Z}=1-92, "$ At. Data Nucl. Data Tables 54, 181-342 (1993). See also the web site wwwcxro.lbl.gov/optical_constants/for updated scattering factor files.

13. D.G. Stearns, "The scattering of X-rays from nonideal multilayer structures," J. Appl. Phys. 65, 491-506 (1989).

14. D.G. Stearns, K.M. Skulina, M. Wall, C.S. Alford, R.M. Bionta, D.M. Makowiecki, E.M. Gullikson, R. Soufli, J.B. Kortright, and J.H. Underwood, "Beryllium-based multilayer structures," Mat. Res. Soc. Symp. Proc. 382, 329-337 (1995).

15. J.H. Underwood, E.M. Gullikson, and K. Nguyen, "Tarnishing of Mo/Si multilayer x-ray mirrors," Appl. Opt. 32, 69856990 (1993).

16. D.G. Stearns, D.P. Gaines, D.W. Sweeney, and E.M. Gullikson, "Nonspecular x-ray scattering in a multilayer-coated imaging system," submited to J. Appl. Phys.

17. E.M. Gullikson, "Nonspecular scattering for normal incidence EUV optics," in these proceedings.

18. P.B. Mirkarimi and C. Montcalm, "Advances in the reduction and compensation of film stress in high-reflectance multilayer coatings for extreme ultraviolet lithography applications," in these proceedings.

19. A.K. Ray-Chaudhuri, S.E. Gianoulakis, P.B. Spence, and M. Kanouff, "Impact of thermal and structural effects on EUV lithographic performance," in these proceedings.

20. R.R. Kola, D.L. Windt, W.K. Waskiewicz, B.E. Weir, R. Hull, G.K. Celler, and C.A. Volkert, "Stress relaxation in Mo/Si multilayer structures," Appl. Phys. Lett. 60, 3120-3122 (1992).

21. D.L. Windt, W.L. Brown, C.A. Volkert, and W.K. Waskiewicz, "Variation in stress with background pressure in sputtered Mo/Si multilayer films," J. Appl. Phys. 78, 2423-2430 (1995).

22. T.D. Nguyen, C. Khan-Malek, and J.H. Underwood, "Achievement of low stress in Mo/Si multilayer mirrors," in Vol. 23 of OSA Proceedings on Extreme Ultraviolet Lithography series, F. Zernike and D.T. Atwood, Eds. (Optical Society of America, Washington, D.C., 1995), pp. 56-59.

23. T.D. Nguyen, "Intrinsic stress and microstructural evolution in sputtered nanometer single and multilayred films," in Polycrystalline Thin Films: Structure, Texture, Properties, and Applications, K. Barmak, M.A. Parker, J.A. Floro, R. Sinclair and D. A. Smith, Eds., Mat. Res. Soc. Symp. Proc. 343, 579-590 (1994).

24. T.D. Nguyen, R. Gronscky, and J.B. Kortright, "Microstructure and stability comparison of nanometer period W/C, WC/C, and Ru/C multilayer structures," in Mater. Res. Soc. Symp. Proc., 187, 95-104 (1990).

25. T.D. Nguyen, X. Lu, and J.H. Underwood, "Stress characterization in periodic multilayer structures for X-ray optics," in Physics of X-Ray Multilayer Structuresin Vol. 6 of 1994 OSA Technical Digest series (Optical Society of America, Washington, D.C., 1994), pp. 103-105.

26. T.D. Nguyen and J.H. Underwood, "Stress, microstructure, and thermal behavior in Mo/Si x-ray multilayer," in Structure and Properties of Multilayered Thin Films, T.D. Nguyen, B.L. Lairson, B.M. Clemens, S.-C. Shin and K. Sato, Eds., Mat. Res. Soc. Symp. Proc. 382, 296-301 (1995).

27. M.C.K. Tinone, T. Haga, and H. Kinoshita, "Multilayer sputter deposition stress control," J. Electron Spectrose Relat. Phenom. 80, 461-464 (1996).

28. M.E. Kassner, F.J. Weber, J. Koike, and R.S. Rosen, "Structural and residual stress changes in Mo/a-Si multilayer thin films with annealing," J. Mat. Sci. 31, 2291-2299 (1996). 
29. R.S. Rosen, M.A. Viliardos, M.E. Kassner, D.G. Stearns, and S.P. Vernon, "Thermal stability of Mo/Si multilayers," in Multilayer Optics for Advanced X-Ray Applications, N.M. Ceglio, Ed., Proc. Soc. Photo-Opt. Instrum. Eng. 1547, $212-$ 220 (1991).

30. D.P. Gaines, R.C. Spitzer, N.M. Ceglio, M. Krumrey, and G. Ulm, "Radiation hardness of molybdenum silicon multilayers designed for use in a soft-X-ray projection lithography system," Appl. Opt. 32, 6991-6998 (1993).

31. D.G. Stearns, R.S. Rosen, and S.P. Vernon, "Fabrication of high-reflectance Mo-Si multilayer mirrors by planar-magnetron sputtering," J. Vac. Sci. Tcchnol. A 9, 2662-2669 (1991).

32. J.E.M. Goldsmith, P.K. Barr, K.W. Berger, L.J. Bernandez, G.F. Cardinale, J.R. Darnold, S.J. Haney, G.C. Henderson, K.J. Jefferson, K.D. Krenz, G.D. Kubiak, R.P. Nissen, Y. Perras, A.K. Ray-Chaudhuri, T.G. Smith, R.H. Stulen, D.A. Tichenor, A.A. Ver Berkmoes, and J.B. Wronosky, "Recent advances in the Sandia EUV 10x microstrepper," in these proceedings.

33. E. Spiller, F.J. Weber, C. Montcalm, S.L. Baker, and E.M. Gullikson, "Multilayer coating and tests of a 10x extreme ultraviolet lithography camera," in these proceedings. 


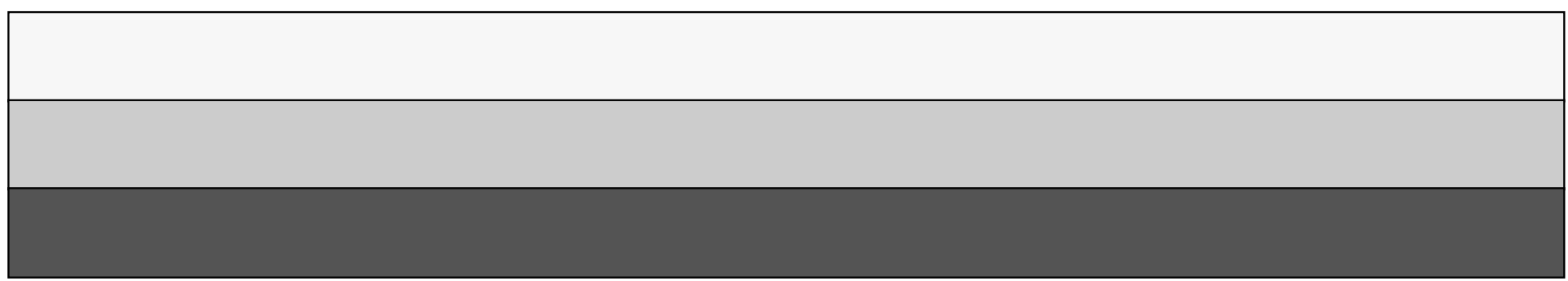

